\title{
Smeared Gauge Fixing
}

\author{
J.E. Hetrick ${ }^{\mathrm{a}}$ and Ph. de Forcrand ${ }^{\mathrm{b}}$ \\ aphysics Department, University of the Pacific, Stockton, CA 95211

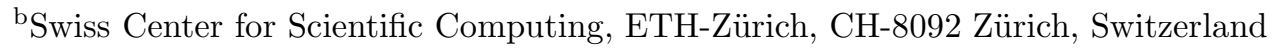

We present a new method of gauge fixing to standard lattice Landau gauge, Max $\operatorname{Re} \operatorname{Tr} \sum_{\mu, x} U_{\mu, x}$, in which the link configuration is recursively smeared; these smeared links are then gauge fixed by standard extremization. The resulting gauge transformation is simultaneously applied to the original links. Following this preconditioning, the links are gauge fixed again as usual. This method is free of Gribov copies, and we find that for physical parameters $(\beta \geq 2$ in $\mathrm{SU}(2))$, it generally results in the gauge fixed configuration with the globally maximal trace. This method is a general technique for finding a unique minimum to global optimization problems.

There are two outstanding difficulties with gauge fixing on the lattice:

1. The Gribov problem: covariant gauge fixing inevitably leads to multiple solutions of the gauge fixing condition.

2. The Smoothness condition: if one alters the gauge condition so that it is free of Gribov copies (like axial gauge) it is generally not smooth, and difficult to compare to perturbation theory.

Below we present a method addressing these two issues which is both simple and fast. It was inspired by the use of improved operators and the smoothing properties of smearing links. It is free of Gribov copies, and usually produces the smoothest $\left(\operatorname{Max} I\left(U_{\mu}, G\right) \equiv \operatorname{Re} \operatorname{Tr} \sum_{x, \mu} U_{\mu}^{G}\right)$ configuration.

It should be contrasted to other covariant solutions to the gauge fixing problem [1], which are also Gribov copy free, but require non-trivial computational resources and do not rotate fields to Landau gauge.

\section{Method}

We begin by iteratively smearing a copy of the links, ie. recursively smearing again the smeared links, with an APE type smearing process. In this study we use the method of [2] in which links are averaged with connecting staples; staples have weight $w$, and the resulting link is reunitarized into $\mathrm{SU}(\mathrm{N})$.

We have found numerically that as we iterate this process there is a critical weight $w_{c}$ below which the configuration is cooled, ie. the average plaquette, moves closer and closer to the identity; with a weight above $w_{c}$ smearing moves the links to a "rough" configuration with $\operatorname{Tr}\langle\square\rangle \sim 0$. The value of this critical weight seems to depend weakly on $\beta$ and volume: $w_{c} \sim 0.5-0.6$.

This method of cooling however is equivariant: If starting configurations $U_{\mu}^{A}$ and $U_{\mu}^{B}$ are related by a gauge transformation $G^{A B}$, then the corresponding smeared configurations $V_{\mu}^{A}$ and $V_{\mu}^{B}$ are related by the same gauge transformation $G^{A B}$. This is useful since the smeared configuration is approaching the trivial orbit, where we know the unique Landau gauge configuration. It is the configuration in which each link is constant and diagonal: $U_{\mu}(x)=\left(D_{\mu}\right)^{1 / n_{\mu}}$ such that $\operatorname{Tr} D_{\mu}=\operatorname{Tr} P_{\mu}$ where $P_{\mu}$ is the smeared Polyakov loop in the $\mu$-direction (made of $n_{\mu}$ links). We neglect here issues of degeneracy of eigenvalues, etc.

Thus if we smear extremely close to the trivial orbit, we can find a $G_{V D}$ which rotates the smeared links $V_{\mu}$ to Landau gauge uniquely, then apply this $G_{V D}$ to the original links $U_{\mu}$, producing a unique configuration $D_{\mu}^{\prime}$; due to the nonlinearity of non-abelian gauge transformations, it is neither diagonal nor satisfies the Landau gauge condition in $\mathrm{SU}(\mathrm{N})$ theory. Still it represents a 
unique starting point on the physical orbit, from which we can gauge fix to Landau gauge and be assured of a unique solution. This is displayed in figure 1.

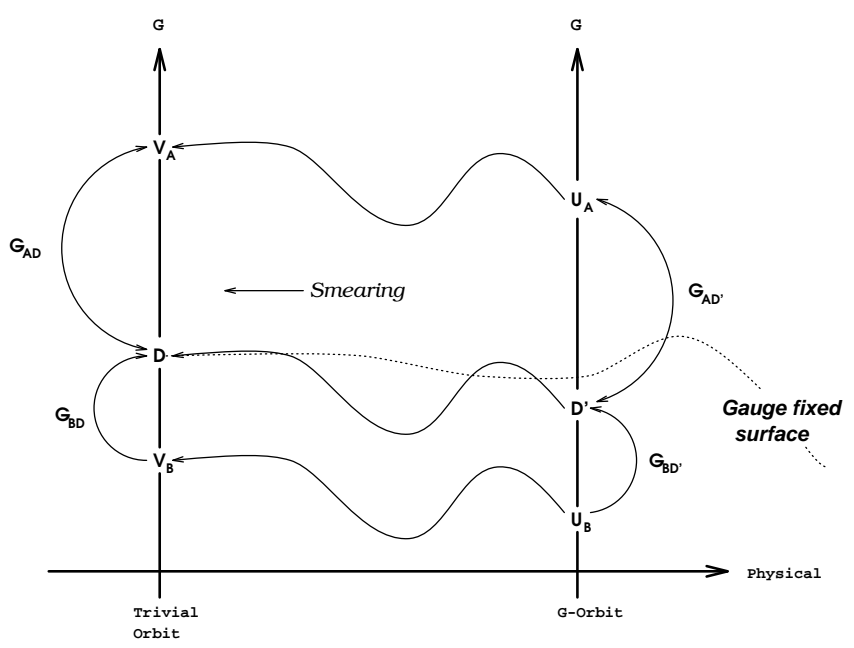

Figure 1. Gauge fix $V_{\mu}$ to $D_{\mu}$ and use the same $G$ to fix $U_{\mu}$ to $D_{\mu}^{\prime}$.

\section{A simpler way}

In our experiments we have found that it is not necessary to smear the links all the way to the trivial orbit; after only a moderate amount of smearing, gauge fixing of the smeared links is unique. Our view of this is captured below in an artist's rendering of the change in $I(V, G)$ as we smear.

If the original configuration is smooth enough, the global maximum on the smeared orbit may lie in the basin of attraction of the (gauge transformed) global maximum on the physical orbit. When this happens, the preconditioning step of gauge fixing the smeared links to Landau gauge leads to the final copy being the one of maximal $I\left(U_{\mu}, G\right)$, ie. the smoothest copy. If the original configuration is quite rough (unphysically so), there often is a mismatch between the global maxima of the smeared and original $I$ functions. The resulting configuration then does not have the globally maximal trace, although the trace is usually still rather high among the copies. In either event though, gauge fixing (to Landau gauge) is unique.

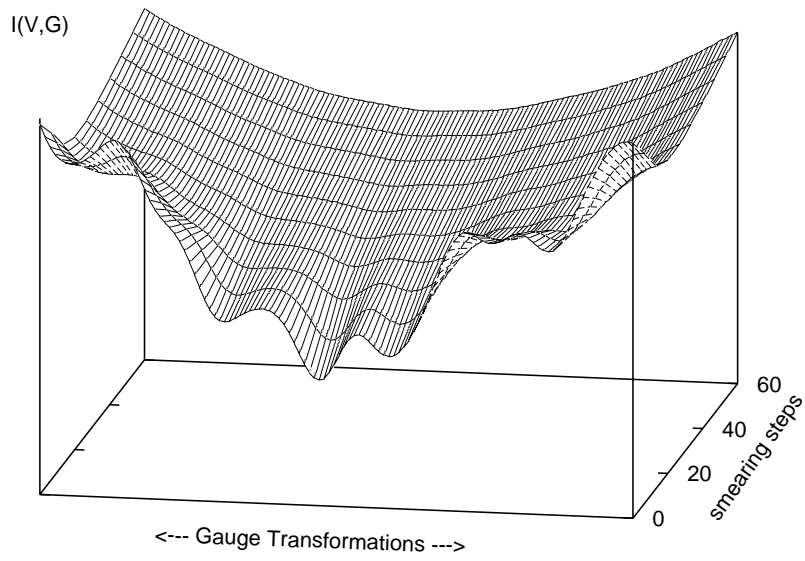

Figure 2. The gauge fixing functional $-I(V, G)$ changes as we smear $V_{\mu}$. Initially the functional has many relative minima leading to Gribov copies. As the links are recursively smeared $-I(V, G)$ becomes smoother, developing a unique minimum.

\section{Parameters}

In the original form of this method, we should smear until we are within machine zero of the trivial orbit $\left(F_{\mu \nu}=0\right)$, however we have found that only partial smearing is necessary. The optimal parameters specifying this have emerged rather heuristically, and we will simply summarize them here and refer the reader to our more complete paper on this method of gauge fixing [3]. Also we have studied small lattices so far, up to $12^{4}$, and we gauge fix to variations smaller that $10^{-13}$ in $I$.

The smearing coefficient $w$, which is the weight given to the staples should be as large as possible, but below the critical value so that the smeared configuration is smoothed. We have found that $w \sim 0.3$ works best, looking at how topological objects (which slow the cooling) are annihilated.

The most important parameter is the number of smearing sweeps done. We have found that this depends on the number of topological objects in the configuration. Thus smearing is pursued until the action falls below the one-instanton bound. On our $8^{4}$ lattices, we smeared until the average plaquette was within $10^{-5}$ of $\mathbf{1}$, which then leads to unique gauge fixing. In figure 3 we show the 
cooling history of 6 lattices at a range of $\beta$ values. The plateaux show the stability of instanton pairs under smearing (like under cooling).

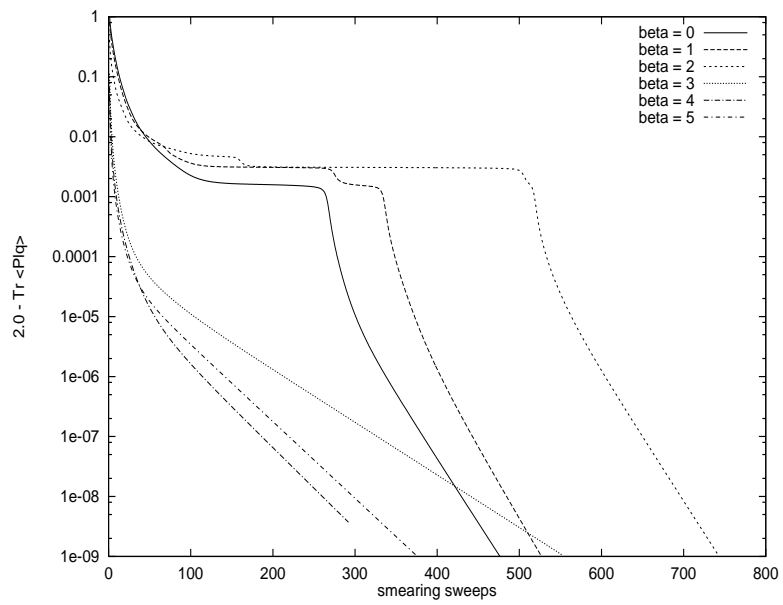

Figure 3. $2.0-\operatorname{Tr}\langle\square\rangle$ versus smearing $(w=0.3)$ on lattices of size $8^{4}$.

\section{Summary}

In figure 4 we display typical results on 100 random gauge transformations at $3 \beta$ values: 2.0 (top), 1.75 (middle), and 1.5 (bottom). The graphs contain histograms of the final value of $I$ on the gauge fixed copies obtained using local extremization, and the arrow shows the unique copy obtained with our method. To summarize our method:

- Recursively smear a configuration $U_{\mu}$ into $V_{\mu}$.

- Gauge fix $V_{\mu}$ by extremizing, $I(V, G) \equiv$ $\sum_{x, \mu} \operatorname{Re} \operatorname{Tr} G^{\dagger}(x) V_{\mu}(x) G(x+\mu)$, and apply the same gauge transformation to $U_{\mu}$.

- Finally, gauge fix $U_{\mu}$ as usual, by extremizing $I(U, G)$.

This construction trivially generalizes to Coulomb or maximal Abelian gauges, and can be more generally applied to some optimization problems.
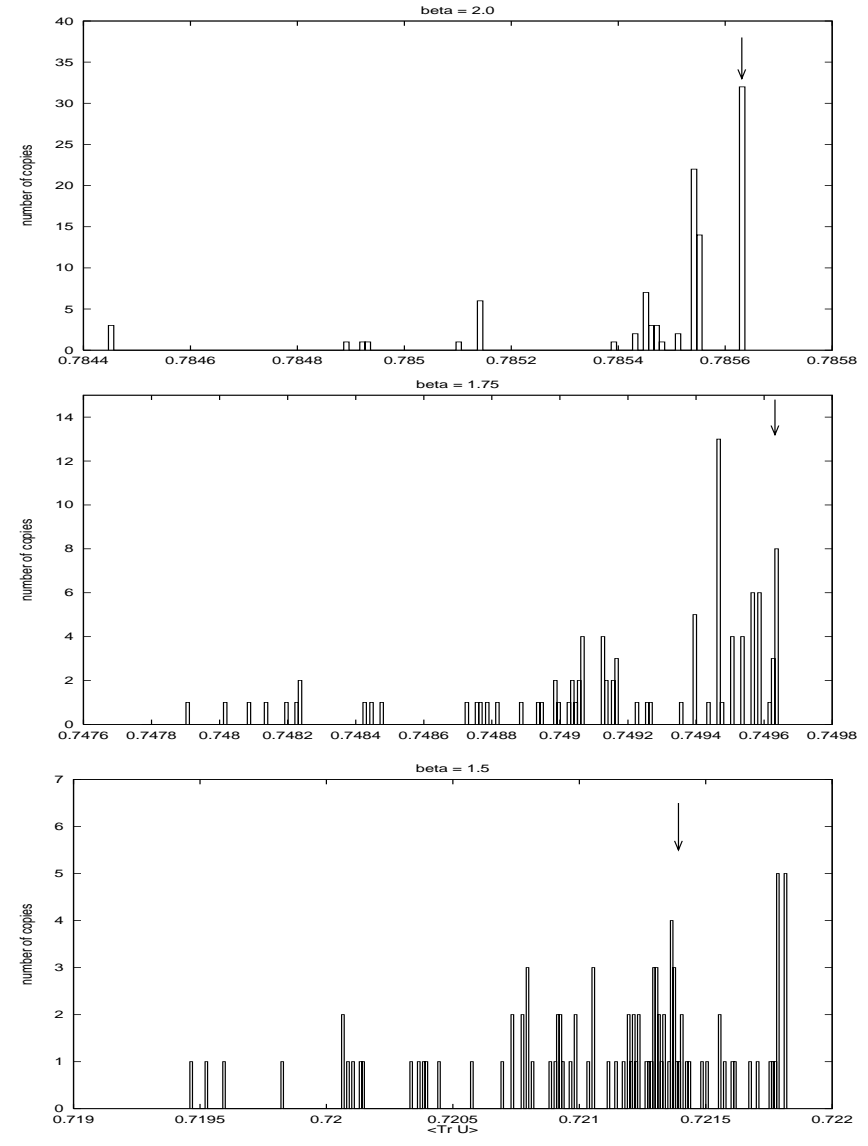

Figure 4. Gribov copies obtained by standard extremization and the unique copy $\downarrow$ obtained from smearing.

\section{REFERENCES}

1. Phys. Lett. B289 (1992) 122

2. Phys. Rev. D55 (1997) 1133

3. in preparation. 